\title{
Temperature-drift instabilities of plasma waves in preflare plasma of solar active regions
}

\author{
A. N. Kryshtal ${ }^{1} \dagger$ and S. V. Gerasimenko ${ }^{1}$ \\ ${ }^{1}$ Main Astronomical Observatory, National Academy of Sciences of Ukraine, \\ email: kryshtal@mao.kiev.ua, geras@mao.kiev.ua
}

A preflare situation in a loop structure for stability to the development of plasma waves at the chromosphere part of a current circuit of a loop is investigated. We consider the situation when plasma has the spatial gradients of the density and temperature. In accordance with Heyvarts-Priest-Rust theory such a preflare situation would typically exist when the amplitude of weak electric field in the circuit of an "old" loop in an active region begins to increase as "new" magnetic flux emerges from under the photosphere (Heyvaerts, Priest \& Rust (1977)). The main physical approximations employed were the following: 1) "weak-field" approximation

$$
\varepsilon_{R}=E_{0} / E_{D}<<1,
$$

where $E_{0}=E_{0}(t)$ is the amplitude of quasi-static electric field in a loop, and $E_{D}$ is the local Dreicer field value; 2) "long-wave" approximation

$$
k_{\perp}^{2} v_{T e}^{2} \cdot \Omega_{e}^{-2} \equiv z_{e}<<z_{i} \equiv k_{\perp}^{2} v_{T i}^{2} \cdot \Omega_{i}^{-2}<<1,
$$

where $v_{T e}$ and $v_{T i}$ are the electron and ion thermal speeds respectively, $\Omega_{e}$ and $\Omega_{i}$ are the electron and ion gyrofrequencies, and $k_{\perp}$ is the perpendicular component of the perturbation wave-vector; 3) "quasi-perpendicular" direction of the propagation

$$
k_{R} \equiv k_{z} / k<<1,
$$

where $k_{z}$ is the longitudinal component of perturbation wave-vector; 4) low-frequency range of perturbations

$$
\begin{gathered}
\omega<<\Omega_{i}, \\
v_{T i}<<\omega / k_{z}<<v_{T e} ;
\end{gathered}
$$

$5)$ the approximation of the local solutions of the dispersion relation, when we can neglect the influence of the boundaries; 6 ) we assumed that reduced spatial gradients of densities $\frac{\partial}{\partial x}\left\{\ln n_{0 e, i}\right\}$ of electrons and ions as well as thats of their temperatures $\frac{\partial}{\partial x}\left\{\ln T_{0 e, i}\right\}$ are the values of the same order, and they dominate in comparison with the corresponding value for magnetic field. Actually, it means that we can neglect the "shear" of magnetic field lines, if the condition

$$
\beta_{i} \equiv 8 \pi n_{0 i} k_{B} T_{0 i} \cdot B_{0}^{-2}<<z_{i} k_{R}^{2}\left(\beta_{A} u_{e}\right)^{-2}
$$

is satisfied. Here $\beta_{A} \equiv v_{T e} / v_{A}$, where $v_{A} \equiv c \Omega_{i} \omega_{P i}^{-1}$ is the Alfven speed, and $\omega_{P i}$ is the ion plasma frequency, and $u_{e} \equiv e E_{0} / m_{e} \nu_{e i}$ is the speed of the electrons with respect to the ions as well $\nu_{e i}$ is the electron-ion collision frequency. The corresponding values of the density and temperature from "MANV equation of state" (Machado, Avrett,Vernazza \&

$\dagger$ Present address: Main Astron. Observ., 27 Akademika Zabolotnoho St., 03680 Kyiv, Ukraine. 
Noyes (1980)) provide the satisfaction of the condition (0.6). With taking into account all these conditions and approximations the dispersion relation (DR) can be simplified and reduced to the algebraic equation of the 4-th order for the reduced frequency $\Omega \equiv \omega / k_{z} v_{A}$. This equation can be solved through the standard Euler method (Van der Waerden(1930)). The analysis of stability of the roots of this DR has given the following results: a) in the case of "weak inhomogeneity" at the most early stage of interaction of the magnetic fluxes, when electron-ion collisions dominate and value of $\varepsilon_{R}$ does not differ greatly from its "steady-state" value $\left(\varepsilon_{R}\right)_{0}$, in the ranges

$$
\begin{gathered}
-0.04 \leqslant k_{R} \leqslant-0.01, \\
0.12 \leqslant z_{i} \leqslant 0.20,
\end{gathered}
$$

there are only two unstable roots of DR, which become such ones only for $t \equiv T_{e} / T_{i}>$ $t_{\text {bound }}=4$ and $\varepsilon_{R}>\left(\varepsilon_{R}\right)_{\text {bound }}=1.01 \times 10^{-4}$. One of these roots, which corresponds to the "modified slow magnetoacoustic-like wave", has the growth rate, which is always positive in the ranges $(0.7) \div(0.8)$. The corresponding instability develops much faster and results in the appearance of anomaleous resistivity. The another one root, which corresponds to the "modified kinetic Alfven wave" (KAW) has the growth rate, which becomes positive only for $z_{i}>\left(z_{i}\right)_{\text {bound }}$ and $k_{R}>\left(k_{R}\right)_{\text {bound }}$. It means that instability under investigation appears and "begins to generate this modified KAW" only at some definite stage of the preflare process development. This can be considered as a "forerunner" of a flare. The negative values of $k_{R}$ in eq.(0.7) mean that the perturbation has Z-component directed to the photosphere. The instabilities employed appear only at

$$
\nabla \equiv \frac{\partial}{\partial x}\left\{\ln n_{0}\right\} / \frac{\partial}{\partial x}\left\{\ln T_{0}\right\}=-3 .
$$

This parameter can be considered as the ratio of characteristic mean scale of temperature to that of the density. Its negative value means that we investigate the loops with the "cool cores" (Foukal(1975)). In the case of "strong inhomogeneity" at much more late stage of the fluxes' interaction, when the collisions of the electrons with saturated ion-acoustic turbulence dominate (Ionson(1978)) and value of $\varepsilon_{R}$ differs greatly from its "steady-state" value $\left(\varepsilon_{R}\right)_{0}$, in the ranges

$$
\begin{gathered}
0.10 \leqslant k_{R} \leqslant 0.20 ; \\
0.10 \leqslant z_{i} \leqslant 0.20
\end{gathered}
$$

the only one unstable root of DR exists. This root corresponds to the "modified slow magnetoacoustic-like wave". Its instability rises also at $\nabla \equiv-3$, but $\left(\varepsilon_{R}\right)_{\text {bound }}=2 \times 10^{-3}$, when $t=15,66$. This last condition provides the possibility of the comparison of our model with model in (Somov, Titov\& Verneta (1987)) for neutral current sheet with saturated ion-acoustic turbulence. In comparison with the case of "weak inhomogeneity" the case of "strong inhomogeneity" evidentely provides much more high probability of the short-time prediction of a flare. In this case due to the sharp increase of a number of the "runaway" electrons the enough strong type-III burst with low-frequency modulation can appear due to the generation of the KAWs or "SMA-like". The "prediction time" in this case essentially decreases (Kryshtal(2002)).

\section{References}

Foukal P. 1975 J. Solar Physics. 43, 327-336.

Heyvaerts,J., Priest,E. \& Rust,D. 1977 Ap.J. 216, 213-221.

Ionson,J. 1978 Ap.J. 226, 650-673.

Kryshtal, A.N. 2002 J.Plasma Phys. 68,part 2, 137-148.

Machado, M., Avrett, E., Vernazza, J. \&Noyes, R. 1980 Ap.J. 242, 336-351.

Somov, B., Titov,V. \&Verneta, A. 1987 Res.Sci.Tech.,Ser.Astron. (USSR) 34, 136-237.

Van der Waerden 1930 Modern Algebra, Springer. 